\title{
Towards High-Order Fluctuation-Splitting Schemes for Navier-Stokes Equations
}

\author{
Hiroaki Nishikawa *and Philip L. Roe ${ }^{\dagger}$ \\ W. M. Keck Foundation Laboratory for Computational Fluid Dynamics \\ Department of Aerospace Engineering \\ The University of Michigan, Ann Arbor, MI 48109
}

\begin{abstract}
This paper reports progress towards high-order fluctuation-splitting schemes for the Navier-Stokes Equations. High-order schemes we examined previously are all based on gradient reconstruction, which may result in undesired mesh-dependency problem due to the somewhat ambiguous gradient reconstruction procedures. Here, we consider schemes for $P_{2}$ elements in order to eliminate the need for such gradient reconstruction. For pure advection, a $P_{2}$ version of the LDA scheme is derived from a constrained least-squares minimization. This scheme is fourth-order accurate. For pure diffusion, a $P_{2}$ Galerkin scheme is derived from a minimization principle, which turns out to be equivalent to applying Richardson's extrapolation technique to the standard second-order Galerkin scheme. This scheme is again fourth-order accurate. Finally, strategies for integrating $P_{2}$ advection and diffusion schemes to develop uniformly accurate $P_{2}$ schemes are discussed.
\end{abstract}

\section{Introduction}

Accurate heat transfer prediction in hypersonic flows requires a CFD code to have excellent performance in resolving various types of flows: strong shocks, almost incompressible flows near stagnation, and viscous layers, especially on unstructured grids. Because of dissatisfaction with current finite-volume codes for this type of problem, ${ }^{1}$ we are trying to develop a radically new methodology for solving the compressible NavierStokes equations. We have been focusing on the fluctuation-splitting schemes as a new method because they are based on multidimensional upwinding and have a better performance on unstructured grids than the conventional finite-volume method. In the previous work, ${ }^{2}$ taking the two-dimensional advection-diffusion equation,

$$
u_{t}+a u_{x}+b u_{y}=\nu\left(u_{x x}+u_{y y}\right) .
$$

as a model equation, we examined the fluctuation-splitting schemes. We suggested reduction to an equivalent first-order system and unified treatment of the advective and the diffusive fluctuations, and then developed third and fourth order schemes based on gradient reconstruction. Such schemes can be readily extended to the Navier-Stokes equations, but there is a concern about the mesh-dependency of the schemes because of the irregular nature of the gradient recovery methods. To eliminate such a concern, we consider here an alternative way to achieve high-order accuracy without reconstructing the solution gradients. The alternative is the use of $P_{2}$ elements which introduce additional degrees of freedom at the midpoints of edges. $P_{2}$ elements have just enough degrees of freedom to represent a solution by a quadratic variation, thereby we do not have to reconstruct anything to better represent the solution. But as the price of this merit, we now need to devise a scheme to compute the solution at the midpoints. In this paper, we present such schemes for a pure advection and a pure diffusion equation. We will show that it is possible to achieve fourth-order accuracy with $P_{2}$ elements without any kind of reconstruction.

\footnotetext{
${ }^{*}$ Research Fellow, Member AIAA

${ }^{\dagger}$ Professor, Fellow AIAA
} 


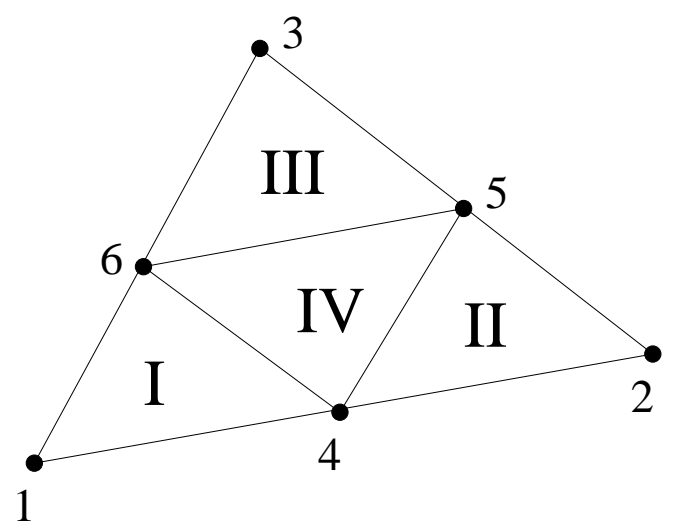

Figure 1. A typical $P_{2}$ element

\section{Fluctuation-Splitting Schemes and Reconstruction}

Fluctuation-splitting schemes are based on nodal variables, leading to cell-based residuals (fluctuations) that are distributed to the nodes. Consider, for example, solving a pure advection equation,

$$
u_{t}+a u_{x}+b u_{y}=0
$$

in a domain divided into a set of triangles $\{T\}$. In a loop over the elements, we compute the fluctuation $\phi_{T}$ for each triangle $T$,

$$
\phi^{T}=\int_{T} u_{t} d x d y=\int_{T}\left[-\left(a u_{x}+b u_{y}\right)\right] d x d y
$$

assuming a piecewise linear variation of $u$, and then distribute it to the nodes of the triangle, thus accumulating the changes at every node. This results in the following update formula for the solution at node $j$

$$
u_{j}^{n+1}=u_{j}^{n}-\frac{\Delta t}{V_{j}} \sum_{T \in\left\{T_{j}\right\}} \phi_{i}^{T}
$$

where

$$
\phi_{i}^{T}=\beta_{j}^{T} \phi^{T}
$$

and $\left\{T_{j}\right\}$ is a set of triangles that shares the node $j, \Delta t=t^{n+1}-t^{n}$ is a (local or global) timestep, $V_{j}$ is the volume of the median dual control volume, and $\beta_{j}^{T}$ is the distribution coefficient that assigns the fraction of the fluctuation sent to the node $j$ within the triangle $T$.

In whatever way the fluctuation is distributed, the method is at most second-order accurate because of the assumption of the linear variation (except for some very special cases). To achieve higher-order, we need to establish high-order representation of the solution within each element and then evaluate the fluctuation with it. Consider a triangular element with midpoints introduced (cf. Figure 1). To evaluate the fluctuation, we convert the integral to a contour integral

$$
\phi_{T}^{H}=\int_{T}\left(a u_{x}+b u_{y}\right) d x d y=\oint_{\partial T}(a u d y-b u d x)
$$

and apply Simpson's rule along each edge. Note that this is fourth-order accurate. Now, instead of storing the solutions at the midpoints as unknowns, we may interpolate the solution. To achieve higher-order, such an interpolation must be better than the linear interpolation. To this end, we recover the gradients at nodes by a least-squares reconstruction technique, and use them to construct the Hermite interpolation along each edge. This gives the midpoint values with sufficient accuracy, and completely determines the high-order fluctuation. 
Note that the midpoints have no further roles. This high-order fluctuation can then be distributed to the three nodes of the triangle. The method just described above is exactly the one proposed by Caraeni and Fuchs $^{3}$ which they claim third-order accurate. Later, Nishikawa, Rad and Roe ${ }^{4}$ rearranged their method so that it can be implemented as adding a high-order correction to existing second-order code. In the previous work, ${ }^{2}$ we confirmed that this type of high-order fluctuation-splitting scheme achieved third-order accuracy with the Green-Gauss gradient reconstruction and fourth-order accuracy with the quadratic least-squares reconstruction. However, these reconstruction schemes do not appear to be grid independent because of the irregularity of the stencil for reconstruction, especially with the quadratic least-squares reconstruction which involves nodes that are not immediate neighbor. In this paper, we consider the alternative, i.e. treat the midpoint values as unknowns and develop schemes directly applied to the quadratic elements. Such schemes are expected to have better accuracy on unstructured grids partly because they preserve polynomial solutions of higher order on arbitrary grids.

\section{III. $\quad P_{2}$ Advection Scheme}

We first consider the advection limit of (1),

$$
u_{t}+a u_{x}+b u_{y}=0 \text {. }
$$

Triangular elements with midpoints are now regarded as $P_{2}$ elements ( See Figure 1). With 6 degrees of freedom $\left\{u_{i}\right\}$ available, we can represent the solution with quadratic variation,

$$
u=\sum_{i=1}^{6} u_{i} N_{i}
$$

where

$$
\begin{aligned}
& N_{1}=\left(2 L_{1}-1\right) L_{1} \\
& N_{2}=\left(2 L_{2}-1\right) L_{2} \\
& N_{3}=\left(2 L_{3}-1\right) L_{3} \\
& N_{4}=4 L_{1} L_{2} \\
& N_{5}=4 L_{2} L_{3} \\
& N_{6}=4 L_{3} L_{1}
\end{aligned}
$$

and $L_{1}, L_{2}$, and $L_{3}$ are the local area coordinates of the triangle. To solve the advection equation, we define the high-order fluctuation as

$$
\phi_{T}^{H}=\int_{T}\left(a u_{x}+b u_{y}\right) d x d y=\oint_{\partial T}(a u d y-b u d x) .
$$

We evaluate this by applying Simpson's rule along each edge. The result can be simplified and written as a sum of second-order fluctuations

$$
\phi_{T}^{H}=\frac{1}{3} \phi_{T}+\frac{8}{3} \phi_{T_{I V}}
$$

where $\phi_{T}$ and $\phi_{T_{I V}}$ are the fluctuations over triangles $T$ and $T_{I V}$ assuming a piecewise linear variation of $u$ within each triangle, i.e.

$$
\begin{aligned}
\phi_{T} & =\sum_{i=1}^{3} k_{i}^{T} u_{i}=\sum_{i=1}^{3}\left[\frac{1}{2}(a, b) \cdot \mathbf{n}_{i}^{T}\right] u_{i} \\
\phi_{T_{I V}} & =\sum_{i=4}^{6} k_{i}^{T_{I V}} u_{i}=\sum_{i=4}^{6}\left[\frac{1}{2}(a, b) \cdot \mathbf{n}_{i}^{T_{I V}}\right] u_{i}
\end{aligned}
$$

where $\mathbf{n}_{i}^{T}$ is a scaled inward normal vector of the edge opposite to the node $i$ in triangle $T$, and similarly for $\mathbf{n}_{i}^{T_{I V}}$. To simplify the notation, we write

$$
\phi_{T}^{H}=\sum_{i=1}^{6} k_{i} u_{i}
$$

$$
3 \text { of } 7
$$




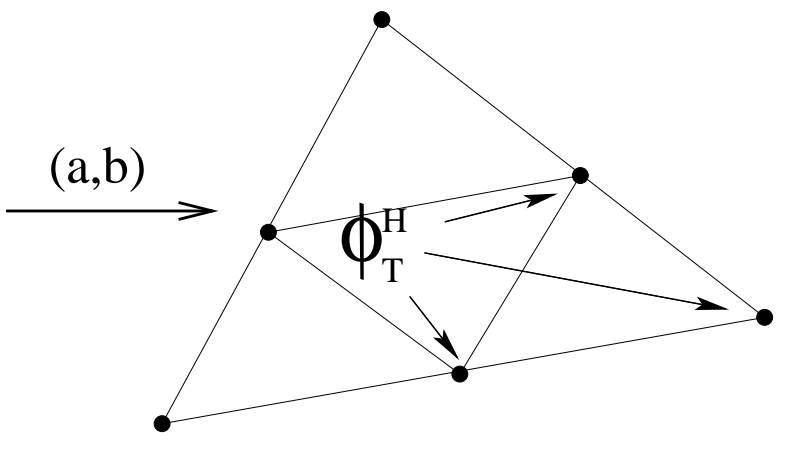

Figure 2. $P_{2}$ LDA Scheme, Case 1

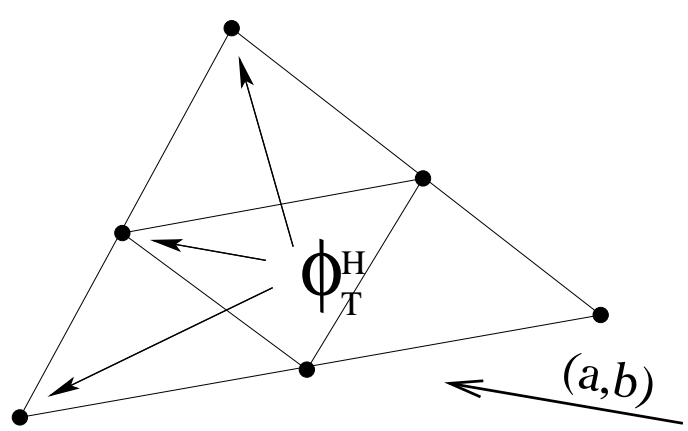

Figure 3. $P_{2}$ LDA Scheme, Case 2

where

$$
k_{i}= \begin{cases}\frac{1}{3} k_{i}^{T} & i=1,2,3 \\ \frac{8}{3} k_{i}^{T_{I V}} & i=4,5,6\end{cases}
$$

To derive a distribution coefficient, we consider minimizing the functional defined by

$$
\mathcal{F}=\frac{1}{2} \sum_{T \in\{T\}}\left(\phi_{T}^{H}\right)^{2} .
$$

Employing the steepest descent method, we obtain the following partial fluctuation $\phi_{j}$, which is distributed to node $j$ within triangle $T$,

$$
\phi_{j}=-\frac{\partial \phi_{T}^{H}}{\partial u_{j}} \phi_{T}^{H}=-k_{j} \phi_{T}^{H}
$$

Unfortunately, this scheme is extremely slow to converge although it is indeed fourth-order accurate. To improve the convergence, we introduce an upwinding constraint and devise the following scheme,

$$
\phi_{j}^{*}=\beta_{j}^{T} \phi_{T}^{H}
$$

where

$$
\beta_{j}^{T}=-\frac{\max \left(0, k_{j}\right)}{\sum_{i=1}^{6} \max \left(0, k_{i}\right)}
$$

i.e., only the downwind nodes get updated (see Figures 2 and 3). Note that the denominator in (24) has been introduced for conservation, i.e. $\sum_{i} \beta_{i}^{T}=1$, and therefore $\sum_{i} \phi_{i}^{*}=\phi_{T}^{H}$. It can be easily shown that exactly the same derivation results in the LDA scheme for $P_{1}$ elements. For this reason, we may call the scheme (24) a $P_{2}$ LDA scheme. Note that the extension to three dimensions is straightforward. Our truncation analysis has confirmed the fourth-order accuracy of this scheme on a regular triangular grid.

The $P_{2}$ LDA scheme was applied to a test problem in a square domain $(0<x<1,0<y<1)$ with the exact solution,

$$
u(x, y)=-\cos (2 \pi(b x-a y))
$$

and $(a, b)=(5,1)$. The solutions were computed for a series of structured and unstructured grids with the number of triangles $800,3200,12800$ in both cases, corresponding to the structured grids of sizes $20 \times 20$, $40 \times 40,80 \times 80$ (see Figures 4 and 5). Structured grids were constructed by inserting a diagonal (bottom-left to upper-right) in a Cartesian cell. The errors are given in Table 1. It is remarkable that the scheme performs very well on unstructured grids, showing the same (or even better) error level especially on fine grids. We remark that, to our knowledge, this is the first fluctuation-splitting scheme for the linear advection equation 


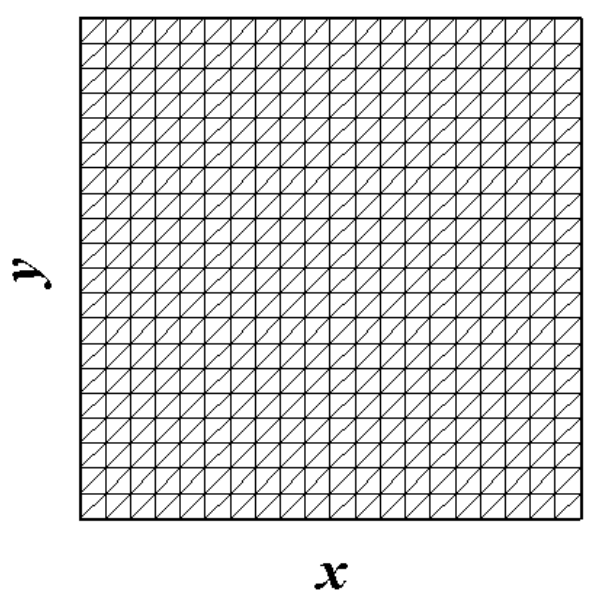

Figure 4. Structured grid (800 triangles)

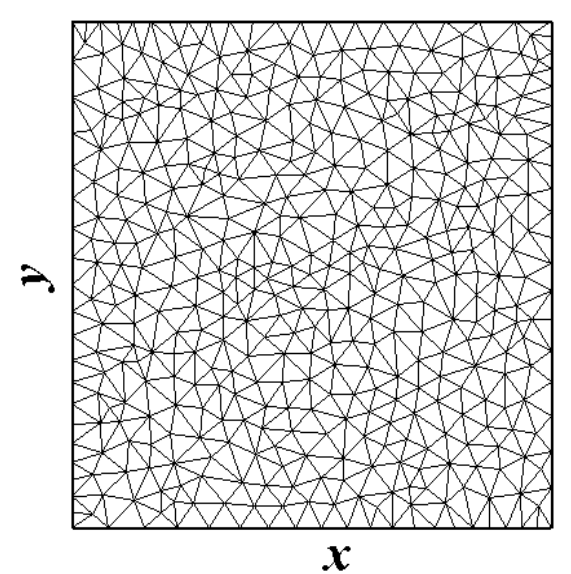

Figure 5. Unstructured grid (800 triangles)

\begin{tabular}{|c|cc|cc|}
\hline & Structured & Order & Unstructured & Order \\
\hline $20 \times 20$ & $6.40 \mathrm{E}-04$ & & $1.00 \mathrm{E}-03$ & \\
$40 \times 40$ & $6.78 \mathrm{E}-05$ & 3.24 & $7.33 \mathrm{E}-05$ & 3.77 \\
$80 \times 80$ & $5.52 \mathrm{E}-06$ & 3.62 & $4.39 \mathrm{E}-06$ & 4.06 \\
\hline
\end{tabular}

Table 1. $P_{2}$ LDA Scheme: $L_{2}$ errors and the order of convergence on structured and unstructured grids.

that has achieved fourth-order accuracy on $P_{2}$ elements. Abgrall and Roe ${ }^{5}$ developed $P_{2}$ schemes based on high-order fluctuations defined on subtriangles, and also Villedieu et. al. ${ }^{6}$ developed $P_{2}$ scheme based on the Petrov-Galerkin formulation of the fluctuation-splitting scheme. But they are all third-order accurate.

It is also possible to devise a scheme by applying the LDA scheme for $P_{1}$ elements to each subtriangle with the high-order fluctuation restricted on that subtriangle. However, we found from a truncation error analysis that it is third-order accurate only when $(a, b)=(2,1)$ (for a particular regular triangular grids mentioned above) and in general only second-order accurate. In fact, the LDA scheme for $P_{1}$ elements also has the same superconvergent property. Therefore, there is basically no improvement with this strategy. We believe that we should distribute the whole high-order fluctuation to each node as all the nodes equally affect the fluctuation. Also, it is important to develop limiters for the $P_{2}$ scheme to achieve both high-order and monotonicity. A type of limiter that is applied to the split fluctuations and hence suitable for $P_{2}$ schemes has been developed by Abgrall and Roe. ${ }^{5}$

\section{IV. $\quad P_{2}$ Diffusion Scheme}

We now turn our attention to the diffusion term and consider solving the Laplace equation with an appropriate time derivative,

$$
u_{t}=u_{x x}+u_{y y}
$$

using the $P_{2}$ elements. It is easy to show by integration by parts that the solution to the Laplace equation minimizes the functional,

$$
\mathcal{F}=\frac{1}{2} \int\left(u_{x}^{2}+u_{y}^{2}\right) d x d y
$$


In fact, the standard $P_{1}$ Galerkin method can be derived by minimizing this on the assumption of the piecewise linear variation of the solution over a triangle. We follow this approach to derive a scheme for $P_{2}$ elements. Using the quadratic variation, we find

$$
\begin{aligned}
\mathcal{F} & =\frac{1}{2} \int\left(u_{x}^{2}+u_{y}^{2}\right) d x d y \\
& =\frac{1}{6} \sum_{T \in\{T\}}\left[\left(u_{x}^{q}\right)^{2}+\left(u_{y}^{q}\right)^{2}\right] S_{T}
\end{aligned}
$$

where $\left(u_{x}^{q}\right)^{2}$ denotes a value expressed by the constant gradients defined in the subtriangles assuming the piecewise linear variation, $u_{x}^{T_{I}}, u_{x}^{T_{I I}}, u_{x}^{T_{I I I}}, u_{x}^{T_{I V}}$,

$$
\begin{aligned}
\left(u_{x}^{q}\right)^{2} & =\frac{1}{4}\left\{\left(u_{x}^{T_{I}}-u_{x}^{T_{I I}}\right)^{2}+\left(u_{x}^{T_{I I}}-u_{x}^{T_{I I I}}\right)^{2}+\left(u_{x}^{T_{I I I}}-u_{x}^{T_{I}}\right)^{2}\right. \\
& \left.+\left(u_{x}^{T_{I}}+u_{x}^{T_{I V}}\right)^{2}+\left(u_{x}^{T_{I I}}+u_{x}^{T_{I V}}\right)^{2}+\left(u_{x}^{T_{I I I}}+u_{x}^{T_{I V}}\right)^{2}\right\}>0
\end{aligned}
$$

similarly for $\left(u_{y}^{q}\right)^{2}$. After further rearrangement, we find that it is possible to rewrite this functional as

$$
\begin{aligned}
\mathcal{F} & =\sum_{T \in\{T\}}\left\{\frac{4}{3} \sum_{T_{\xi} \in\left\{T_{\xi}\right\}} F_{T_{\xi}}-\frac{1}{3} F_{T}\right\} \\
& =\frac{4}{3} \sum_{T \in\{T\}} \sum_{T_{\xi} \in\left\{T_{\xi}\right\}} F_{T_{\xi}}-\frac{1}{3} \sum_{T \in\{T\}} F_{T}
\end{aligned}
$$

where $\left\{T_{\xi}\right\}=\left\{T_{I}, T_{I I}, T_{I I I}, T_{I V}\right\}$, and

$$
\begin{aligned}
F_{T} & =\frac{1}{2}\left[\left(u_{x}^{T}\right)^{2}+\left(u_{y}^{T}\right)^{2}\right] S_{T} \\
F_{T_{\xi}} & =\frac{1}{2}\left[\left(u_{x}^{T_{\xi}}\right)^{2}+\left(u_{y}^{T_{\xi}}\right)^{2}\right] S_{T_{\xi}}
\end{aligned}
$$

where $\left(u_{x}^{T}, u_{y}^{T}\right)$ is the constant gradient on the triangle $T$. It is clear now that the minimizing $F_{T}$ gives the second-order Galerkin scheme for the triangle $T$ while minimizing $F_{T_{\xi}}$ gives the second-order Galerkin scheme for the subtriangles. We thus obtain the following scheme from minimization: four-third of the second-order update on each subtriangle,

$$
u_{j}^{n+1}=u_{j}^{n}-\frac{4}{3}\left\{\frac{\omega}{2}\left(u_{x}^{T_{\xi}}, u_{y}^{T_{\xi}}\right) \cdot \mathbf{n}_{j}^{T_{\xi}}\right\} \quad j \in\left\{i_{T_{\xi}}\right\}
$$

followed by subtracting one-third of the second-order update on the original triangle,

$$
u_{j}^{n+1}=u_{j}^{n}+\frac{1}{3}\left\{\frac{\omega}{2}\left(u_{x}^{T}, u_{y}^{T}\right) \cdot \mathbf{n}_{j}^{T}\right\} \quad j \in\left\{i_{T}\right\}
$$

where $\omega$ is a small constant and $\left\{i_{T_{\xi}}\right\}$ is the set of nodes of the subtriangle $T_{\xi}$. Note that this is nothing but the Richardson extrapolation applied to the 2nd-order Galerkin method. In fact, the weights, $4 / 3$ and $-1 / 3$ could have been found readily from this viewpoint. It is then immediate from this that the resulting scheme is in general third-order accurate. However, the Galerkin scheme does not contain odd order terms in its truncation error at least on regular grids due to the symmetry of its stencil, and so in such a case the method becomes fourth-order accurate. Naturally, we may call this scheme a $P_{2}$ Galerkin scheme.

To demonstrate its accuracy, we consider a test problem in the square domain $(0<x<1,0<y<1)$ with the exact solution,

$$
u(x, y)=\frac{\sinh (\pi x) \sin (\pi y)+\sinh (\pi y) \sin (\pi x)}{\sinh (\pi)} .
$$

Both structured and unstructured grids were used with the number of triangles 200, 800, 3200, corresponding to the structured grids of sizes $10 \times 10,20 \times 20,40 \times 40$. Results are shown in Table 2 . The method is fourth-order accurate on structured grids as we expected. For unstructured grids, it becomes third-order accurate (also as we expected), but the actual errors are comparable to those on structured grids. 


\begin{tabular}{|c|cc|cc|}
\hline & Structured & Order & Unstructured & Order \\
\hline $10 \times 10$ & $2.28 \mathrm{E}-05$ & & $3.22 \mathrm{E}-05$ & \\
$20 \times 20$ & $1.43 \mathrm{E}-06$ & 4.00 & $3.86 \mathrm{E}-06$ & 3.10 \\
$40 \times 40$ & $8.87 \mathrm{E}-08$ & 4.01 & $4.49 \mathrm{E}-07$ & 3.11 \\
\hline
\end{tabular}

Table 2. $L_{2}$ errors and the order of convergence on structured and unstructured grids.

\section{Concluding Remarks}

Obviously, simply adding the two schemes developed here will not yield a uniformly accurate scheme for the advection-diffusion equation: the scheme is fourth-order accurate in both advective and diffusive limits but not in between due to the incompatibility of the discretization methods. ${ }^{2}$ Note in particular that there are no quantities corresponding the fluctuation in the $P_{2}$ Galerkin scheme as the updates sum up to zero over the nodes of the element. In the case of reconstruction schemes, we avoided this problem by solving the equivalent first-order system

$$
\begin{aligned}
u_{t}+a u_{x}+b u_{y} & =\nu\left(p_{x}+q_{y}\right) \\
p-u_{x} & =0 \\
q-u_{y} & =0
\end{aligned}
$$

and achieve uniformly high-order accuracy. The same could be done for $P_{2}$ elements, of course. However, so far, we have not been able to do so due to the difficulty of solving (40) and (41) with sufficiently high order. Another possibility would be to recover the gradients at nodes by some high-order interpolation. This is based on the observation that we need a continuous representation of the gradient in order to define a conservative fluctuation for the diffusion term. Yet another possibility is to introduce the jump in the normal derivative on the element boundary to achieve the gradient continuity as typically done in the finite-element methods.

Further development of the $P_{2}$ advection scheme is also currently underway, focusing on monotonicity preservation.

\section{Acknowledgments}

This work has been sponsored by the Space Vehicle Technology Institute, under grant NCC3-989, one of the NASA University Institutes, with joint sponsorship from the Department of Defense. Appreciation is expressed to Claudia Meyer, Mark Klemm and Harry Cikanek of the NASA Glenn Research Center, and to Dr. John Schmisseur and Dr. Walter Jones of the Air Force Office of Scientific Research.

\section{References}

\footnotetext{
1 "High Energy Flow Solver Synthesis (LAURA Benchmarks: Cylinder)," http://hefss.larc.nasa.gov/index.cfm? selection=laura\&laura_type=benchmarks.

${ }^{2}$ Nishikawa, H. and Roe, P., "A Third-Order Fluctuation-Splitting Scheme That Preserves Potential Flow," Third International Conference on Computational Fluid Dynamics, Toronto, CA, 2004.

${ }^{3}$ Caraeni, D. and Fuchs, L., "Compact Third-Order Multidimensional Upwind Scheme for Navier-Stokes Simulations," Theoretical and Computational Fluid Dynamics, Vol. 15, 2002, pp. 373-401.

${ }^{4}$ Nishikawa, H., Rad, M., and Roe, P., "A Third-Order Fluctuation-Splitting Scheme That Preserves Potential Flow," 15th AIAA Computational Fluid Dynamics Conference, AIAA Paper 01-2595, Anaheim, 2001.

${ }^{5}$ Abgrall, R. and Roe, P. L., "High-Order Fluctuation Schemes on Triangular Meshes," Journal of Scientific Computing, Vol. 19, No. 1-3, 2002, pp. 3-36.

${ }^{6}$ Villedieu, N., Ricchiutoa, M., and Deconinck, H., "Study of a 3rd Order RD Scheme for Advection-Diffusion Equation," VKI Internal Report, 2004.
} 